\title{
CLASSIFICAÇÃO DE GENÓTIPOS DE AMENDOIM BASEADA NOS DESCRITORES AGROMORFOLÓGICOS E ISOENZIMÁTICOS
}

\section{CLASSIFICATION OF PEANUT GENOTYPES BASED ON AGROMORPHOLOGICAL AND ISOENZIMATIC DESCRIPTORS}

\author{
Roseane Cavalcanti dos Santos ${ }^{1}$, José de Alencar Nunes Moreira ${ }^{1}$, \\ Ricardo Henrique de Farias ${ }^{2}$, Jair Moura Duarte ${ }^{3}$
}

\section{RESUMO}

Procedeu-se à classificação de genótipos intraespecíficos de amendoim quanto aos descritores agromorfológicos e isoenzimáticos com auxílio da análise dos componentes principais. Vinte e três descritores foram analisados, dentre esses, onze foram morfológicos, nove agronômicos e três protéico-enzimáticos. Baseado nas evidências da análise dos componentes principais, verificou-se que os principais descritores morfológicos foram o tipo botânico, pigmentação da haste principal, padrão de inflorescência, ponto de maturação da vagem, cor dos folíolos, tamanho da vagem, hábito de crescimento, cor da semente e pilosidade da planta. Nos agronômicos, as maiores cargas foram para o início da floração, peso de 100 sementes, número de vagens/planta, porcentagem de vagens chochas $e$ rendimento em amêndoas. Na combinação desses caracteres, as maiores cargas foram observadas para os descritores porcentagem de vagens chochas, número de semente/vagem, número de vagens/planta, rendimento em amêndoas, tamanho da vagem e o tipo botânico. Na análise dos sistemas enzimáticos, verificou-se que nenhum dos sistemas apresentou qualquer relação entre os padrões de banda e os tipos botânicos; contudo, constatou-se que a similaridade baseada nos tipos agromorfológicos dos genótipos do tipo Valência deve corresponder com a encontrada na base dos sistemas protéico-enzimáticos.

Palavras-chave: Arachis hypogaea, germoplasma, componentes principais, eletroforese.

\section{SUMMARY}

The agromorphological and isoenzimatic descriptors were used to classify peanut infraespecific genotype by principal components analysis. Twenty three descriptors were utilized, among them, eleven were morphologic, nine agronomic and three molecular ones. Based on the results of principal components analysis, it was verified that the main morphological descriptors were botany type, pigmentation of main stem, inflorescence pattern, pod maturation, leaflets colour, growth habit, seed colour and hairiness; the main agronomic descriptors were blomming, 100 seeds weigh, number of pods/plant, 'pops' and seeds yield. As to combined morphological and agronomic descriptors it was verified that 'pops', number of seeds/pod, number of pods/plant, seeds yield, pod size and botany type gave greatest contribution. In relation to molecular analysis, no relation was verified between band patterns and botany types of the genotypes, however, it was reported that the similarity based on the agromorphological descriptors of the Valencia genotypes should correspond to that one found on pattern of the molecular systems.

Key words: Arachis hypogaea, germplasm, principal components, electrophoresis.

\section{INTRODUÇÃO}

O amendoim é uma planta alotetraplóide que se reproduz quase que exclusivamente por autogamia. As práticas das seleções convencionais utilizadas nas populações intra-específicas com essa oleaginosa demandam mais critério e atenção devido às grandes semelhanças fenotípicas que são freqüentemente registradas entre os genótipos. Os trabalhos sobre classificação de acessos utilizando os recursos da análise multivariada, embora ainda escassos, têm oferecido contribuições efetivas na discriminação de genótipos que podem ser potencialmente utilizados no melhoramento dessa oleaginosa, inclusive com indicação dos caracteres mais responsivos para a obtenção de populações geneticamente divergentes.

\footnotetext{
${ }^{1}$ Engenheiro Agrônomo, Embrapa-Algodão, CP 174, 58107-720, Campina Grande, PB. E-mail: roseane@cenargen.embrapa.br.

${ }^{2}$ Estudante de Agronomia, Estagiário Embrapa-Algodão.

${ }^{3}$ Engenheiro Agrônomo, Doutorando em Biotecnologia, UFLA, Lavras, MG. 
ESQUIVEL $\boldsymbol{e t}$ al. (1993 a) estudaram 130 acessos de amendoim, pela análise dos componentes principais, os quais foram caracterizados através de vários descritores quantitativos e qualitativos. Os resultados encontrados confirmaram a classificação taxonômica sugerida por KRAPOVICKAS (1968), concluindo que o uso de métodos multivariados permite o estudo de diversos acessos, usando-se para isso uma lista mínima de descritores. Em outro trabalho, ESQUIVEL et al. (1993b) procederam análise dos componentes principais usando caracteres agronômicos, bioquímicos, morfológicos e fitopatológicos. Os autores verificaram que a distinção entre os tipos botânicos Valência, Spanish (ambos de hábito de crescimento ereto) e Virgínia (hábito ramador) não foi muito clara quando foram utilizados apenas os caracteres quantitativos. Os acessos do tipo Spanish, entretanto, mostraram-se intermediários entre os tipos botânicos Valência e Virgínia. MEHNDIRATTA et $\boldsymbol{a l}$. (1970) afirmam que existe uma série de fatores responsáveis pelo agrupamento diferencial de cultivares, sendo os principais as diferentes arquiteturas genéticas do germoplasma originado de um mesmo local; suas características similares, embora originados de outros locais; e a troca das características dos genótipos com a mesma origem geográfica, devido às pressões de seleção.

No presente trabalho, foi procedida a classificação de vinte genótipos de amendoim de três subespécies diferentes na base dos descritores morfológicos, agronômicos e enzimáticos, com o auxílio da técnica dos componentes principais, objetivando verificar a aplicabilidade dessa técnica na seleção de descritores para o melhoramento dessa oleaginosa.

\section{MATERIAL E MÉTODOS}

Vinte genótipos de amendoim foram cultivados no período das águas em Campina GrandePB durante os anos de 1993 a 1995, plantados no espaçamento de $0,70 \times 0,20 \mathrm{~m}$ e densidade de 10 plantas/metro linear. A parcela foi constituída por três fileiras de $6 \mathrm{~m}$ de comprimento $\left(12,60 \mathrm{~m}^{2}\right)$, tomando-se a fileira central como área útil, desprezando-se $0,50 \mathrm{~m}$ de cada extremidade $\left(3,5 \mathrm{~m}^{2}\right)$. Adotouse o delineamento de blocos ao acaso com três repetições. Correção e adubação química foram procedidas na área experimental de acordo com as recomendações da análise da amostra do solo. A relação dos genótipos econtra-se na tabela 1.

Os descritores avaliados foram: peso de 100 vagens e de 100 sementes, início da floração, percentagem de sementes perfeitas e de vagens chochas, número de vagens/planta, rendimento em sementes e em vagens, colheita, tipo botânico, hábito de crescimento, altura da haste principal, pigmentação da haste, padrão de inflorescência, pilosidade da
Tabela 1 - Tipo botânico e procedência dos genótipos de amendoim. Campina Grande, 1997.

$\begin{array}{lll}\text { Tipo botânico } & \text { Genótipos } & \text { Procedência } \\ & & \\ \text { Valência } & \text { CNPA Roxo } & \text { Sapé - PB } \\ & \text { IAC Tupã } & \text { São Paulo- SP } \\ & \text { Tatu } & \text { São Paulo - SP } \\ & \text { CNPA 95 AM } & \text { Mogeiro - PB } \\ & \text { CNPA 96 AM } & \text { Itabaiana - PB } \\ & \text { CNPA 05 AM } & \text { Salvador - BA } \\ & \text { CNPA 28 AM } & \text { Mangueira - CE } \\ & \text { CNPA 55 AM } & \text { Feira Grande - AL } \\ \text { Spanish } & \text { CNPA 106 AM } & \text { Mogeiro - PB } \\ & \text { CNPA 75 AM } & \text { São Paulo - SP } \\ & \text { CNPA 16 AM } & \text { África do Sul - África } \\ & \text { CNPA 01 AM } & \text { Teresina - PI } \\ & \text { CNPA 76 AM } & \text { Senegal - África } \\ & \text { CNPA 113 AM } & \text { Flórida - EUA } \\ & \text { CNPA 09 AM } & \text { Alagoinha - PB } \\ \text { CNPA 52 AM } & \text { Sapé - PB } \\ \text { Virgínia } & \text { CNPA 125 AM } & \text { Carolina do Norte - EUA } \\ & \text { CNPA 126 AM } & \text { Paraguai } \\ & \text { CNPA 129 AM } & \text { Paraguai } \\ & \text { CNPA 112 AM } & \text { Penápolis - SP } \\ & & \end{array}$

Fonte: Santos et al. (1997b).

planta, cor dos folíolos, cor da semente, número de sementes/vagem, tamanho da vagem e ponto de maturação da vagem. Para classificar os genótipos foi utilizada a análise dos componentes principais calculados a partir da matriz de variância e covariância, segundo metodologia descrita por MOREIRA et al. (1994); essas foram obtidas considerando-se, separadamente, os descritores morfológicos, a média dos descritores agronômicos e a combinação de ambos, para observar o grau de discriminação dessas variáveis na classificação intraespecífica dos genótipos. A classificação foi procedida através do exame da dispersão dos pontos correpondentes aos três tipos botânicos no gráfico formado pelos dois primeiros componentes principais.

Para o estudo das isoenzimas utilizaramse tecidos de semente, sendo empregado o gel de poliacrilamida $(7 \%)$ como meio suporte. Os descritores utilizados foram: proteínas totais (PT), esterase (EST) e fosfatase ácida (ACP). A metodologia de extração e coloração seguiu de acordo com SANTOS et al. (1997a). Na análise estatística desses sistemas, cada banda foi tomada como variável qualitativa e identificada como 1 , quando presente e 0 , no caso de ausente. A seguir, foi adotada a análise dos componentes principais a partir da matriz de variância e covariância com os valores de 1 e 0 , respectivamente, para a presença e ausência das bandas nos vários genótipos (MOREIRA et al. 1994). 


\section{RESULTADOS E DISCUSSÃO}

A tabela 2 contém os autovetores e as percentagens da variância acumulada para os dois primeiros componentes principais, obtidos para os descritores morfológicos, agronômicos e para a soma dos descritores.

Para os descritores morfológicos, os dois primeiros componentes englobaram $73 \%$ da variação total e, por essa razão, foram escolhidos para representar as novas variáveis a partir dos dois primeiros autovetores (tabela 2). As maiores cargas foram detectadas para os descritores tipo botânico, pigmentação da haste principal, padrão de inflorescência, cor dos folíolos, maturação e tamanho das vagens no primeiro componente e hábito de crescimento, cor da semente e pilosidade da planta no segundo componente principal. Esses dois componentes, portanto, foram dominados por descritores

Tabela 2 - Autovetores e percentagens da variância acumulada para o primeiro (C1) e segundo (C2) componente principal para os descritores morfológicos, agronômicos e combinados.

\begin{tabular}{|c|c|c|c|c|c|c|}
\hline \multirow[t]{3}{*}{ Variáveis } & \multicolumn{6}{|c|}{ Descritores } \\
\hline & \multicolumn{2}{|c|}{ Morfológicos } & \multicolumn{2}{|c|}{ Agronômicos } & \multicolumn{2}{|c|}{ Combinados } \\
\hline & $\mathrm{C} 1$ & $\mathrm{C} 2$ & $\mathrm{C} 1$ & $\mathrm{C} 2$ & $\mathrm{C} 1$ & $\mathrm{C} 2$ \\
\hline Tipo botânico & $-0,380$ & $-0,027$ & & & $-0,042$ & $-0,333$ \\
\hline Hábito de crescimento & $-0,236$ & $-0,418$ & & & 0,221 & $-0,229$ \\
\hline Cor da semente & $-0,247$ & 0,406 & & & $-0,262$ & $-0,191$ \\
\hline $\mathrm{N}^{\mathrm{o}}$ semente/vagem & 0,305 & $-0,312$ & & & 0,318 & 0,231 \\
\hline Altura da haste principal $(\mathrm{cm})$ & 0,267 & 0,204 & & & 0,124 & 0,211 \\
\hline Pigmentação da haste & 0,339 & 0,111 & & & 0,112 & 0,289 \\
\hline Padrão de inflorescência & 0,339 & 0,270 & & & $-0,041$ & 0,298 \\
\hline Pilosidade & $-0,151$ & 0,392 & & & $-0,194$ & $-0,09$ \\
\hline Cor dos folíolos & $-0,327$ & $-0,364$ & & & 0,101 & $-0,288$ \\
\hline Ponto de maturação das vagens & $-0,334$ & $-0,109$ & & & $-0,050$ & $-0,270$ \\
\hline Tamanho da vagem & 0,319 & $-0,369$ & & & 0,307 & 0,245 \\
\hline Peso de 100 vagens (g) & & & $-0,333$ & $-0,372$ & 0,269 & 0,135 \\
\hline Peso de 100 sementes $(\mathrm{g})$ & & & $-0,156$ & 0,426 & 0,270 & $-0,223$ \\
\hline Rendimento em sementes $(\mathrm{kg} / \mathrm{ha})$ & & & $-0,166$ & $-0,419$ & $-0,309$ & 0,160 \\
\hline Início da floração ${ }^{1}$ & & & $-0,662$ & 0,063 & 0,254 & $-0,238$ \\
\hline $\mathrm{N}^{\mathrm{o}}$ vagem/planta & & & 0,001 & $-0,443$ & $-0,286$ & $-0,178$ \\
\hline Colheita $^{1}$ & & & 0,320 & 0,181 & 0,212 & $-0,266$ \\
\hline Rendimento em vagens (kg/ha) & & & 0,043 & 0,272 & $-0,060$ & 0,140 \\
\hline Vagem chocha $(\%)$ & & & 0,059 & $-0,441$ & 0,381 & $-0,130$ \\
\hline Semente perfeita $(\%)$ & & & 0,328 & 0,002 & $-0,149$ & 0,104 \\
\hline
\end{tabular}

Variância acumulada (\%)

$54,60 \quad 73,00 \quad 42,33 \quad 62,66 \quad 39,80 \quad 57,60$

\footnotetext{
'Tias após a emergência.
}

que têm, de fato, grande peso na classificação intraespecífica no amendoim.

No caso dos descritores agronômicos, os dois primeiros componentes principais englobaram $62,66 \%$ da variação total, bem menor do que o encontrado para os caracteres morfológicos (tabela 2). Mesmo assim, esses componentes foram escolhidos para representar as novas variáveis a partir dos dois primeiros autovetores. Para estas características, observa-se que as maiores cargas foram para o início da floração no primeiro componente, e peso de 100 sementes, número de vagens/planta, percentagem de vagens chochas e rendimento em sementes no segundo componente principal.

A combinação dos dois tipos de descritores apresentou, para os dois primeiros componentes principais, percentual de apenas $57,66 \%$, valor esse inferior ao observado para os tipos de descritores tomados individualmente. A exemplo dos casos anteriores, esses dois componentes foram, também, tomados para representar as novas variáveis a partir dos dois primeiros autovetores (tabela 2). Na combinação desses caracteres, as maiores cargas foram observadas para os descritores percentagem de vagens chochas, número de semente/vagem, rendimento em sementes, tamanho da vagem e número de vagem/planta no primeiro componente, enquanto que o segundo foi dominado pelo tipo botânico (tabela 2). O primeiro componente, portanto, representou praticamente o eixo da variação para os descritores agronômicos, e o segundo, o correspondente aos caracteres morfológicos.

As distribuições dos pontos correspondentes aos três tipos botânicos no sistema de eixos representando o primeiro e segundo componentes principais, respectivamente, para os caracteres morfológicos, agronômicos e a combinação de ambos caracteres encontram-se nas figuras 1,2 e 3 . Para os descritores morfológicos foi denotada a presença de três aglomerados englobando os tipos Valência, Virgínia e Spanish (figura 1). Observa-se que a distribuição espacial dos pontos nessa figura não foi uniforme, variou dependendo do tipo botânico considerado; nos tipos Valência e Virgínia, a distribuição foi muito mais concentrada do que nos genótipos do tipo Spanish (figura 1). O resultado dessa distribuição pode ser um indicativo da 


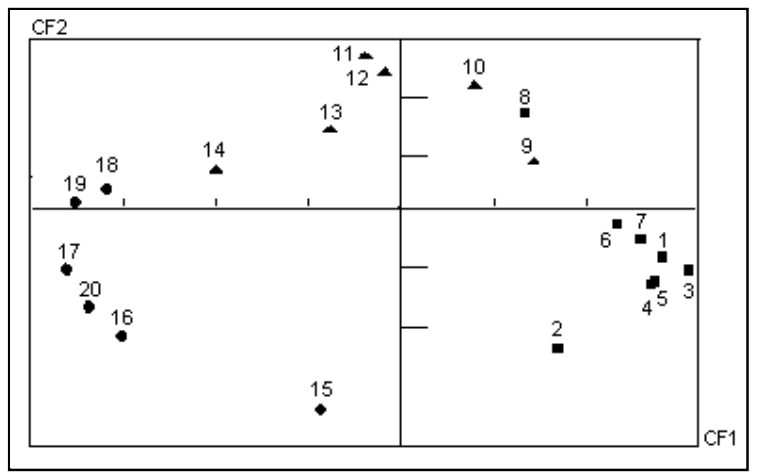

Figura 1 - Distribuição dos pontos correspondentes aos genótipos no plano definido pelos dois primeiros componentes principais para os descritores morfológicos.

Legenda: Virgínia $\bigcirc$ Spanish $\triangle$ Valência

maior estabilidade morfológica dos tipos Valência e Virgínia, comparativamente, aos genótipos pertencentes ao tipo Spanish. ESQUIVEL et al. (1993a), utilizando a análise fatorial de correspondência, conseguiram separar claramente esses tipos botânicos, a partir dos descritores morfológicos previamente selecionados.

Com respeito aos descritores agronômicos, a distinção dos aglomerados não foi tão nítida como no caso dos caracteres morfológicos (figura 2). Mesmo assim, há uma tendência maior de conglomerados dos pontos, representando os tipos Valência, o que, aliás, foi observado também quando foram utilizados os caracteres morfológicos como descritores. A maior dispersão, no caso, ocorreu nos tipos Virgínia e Spanish, para os quais não se evidenciou qualquer tendência de agrupamento na base dos descritores agronômicos adotados (figura 2). Os tipos Valência, portanto, foram bem discriminados tanto para os caracteres morfológicos quanto para os agronômicos. No trabalho de ESQUIVEL $\boldsymbol{e t}$ al. (1993a, b), a despeito dessa ocorrência não ter sido assinalada, observou-se no gráfico com os componentes principais para os descritores quantitativos que a dispersão dos pontos referentes aos tipos botânicos Virgínia e Spanish foi muito mais espalhada do que no caso dos tipos Valência. A explicação para a dispersão observada nos outros grupos pode ser creditada ao fato de que os tipos Virgínia e Spanish são mais amplamente cultivados em nível mundial, como na Índia e nos EUA, por exemplo; muitas de suas características agronômicas são resultantes de hibridações dirigidas, até com outros tipos, visando ao aprimoramento dos cultivares pertencentes a esses dois tipos botânicos para atender exigências específicas de um programa de melhoramento.
Para os descritores combinados, observou-se que os genótipos distribuíram-se claramente em três grupos. Na figura 3, verifica-se que os tipos botânicos acham-se bem delimitados, com os genótipos do tipo Spanish ocupando posição intermediária entre os Valência e os Virgínia. Dessa forma, a combinação dos dois tipos de descritores, morfológicos e agronômicos, ao que parece, compensou a deficiência de cada um deles de modo a melhorar a eficiência na discriminação dos tipos em estudo.

$\mathrm{Na}$ análise dos sistemas enzimáticos, foram encontrados 6, 18 e 10 padrões eletroforéticos para EST, PT e ACP, respectivamente. Em nenhum desses sistemas, foi observada qualquer relação entre os padrões de banda e os tipos botânicos. Em certas situações, como no caso das proteínas totais, foi tanta a variabilidade observada entre os genótipos que, praticamente, registrou-se um padrão para cada germoplasma estudado. Nessas condições, adotou-se a análise dos componentes principais para se observar a possibilidade de grupar os genótipos com base nos padrões de banda.

A partir dos resultados da análise, observou-se que os dois primeiros componentes englobaram $64 \%$ da variação total e, assim, foram tomados para representar as novas variáveis a partir dos dois primeiros componentes principais. A dispersão dos pontos correspondentes nos diversos genótipos no plano formado com esses componentes encontra-se na figura 4. Duas tendências de comportamento podem ser observadas na distribuição desses pontos. A primeira, representada pela concentração dos pontos correpondentes aos tipos Valência, e a segunda, pela dispersão dos referentes aos outros tipos; esse comportamento foi observado, também, para os descritores agronômicos anteriomente estudados. Essa constatação é indicativa de que, pelo menos, para o tipo Valência, a similaridade baseada

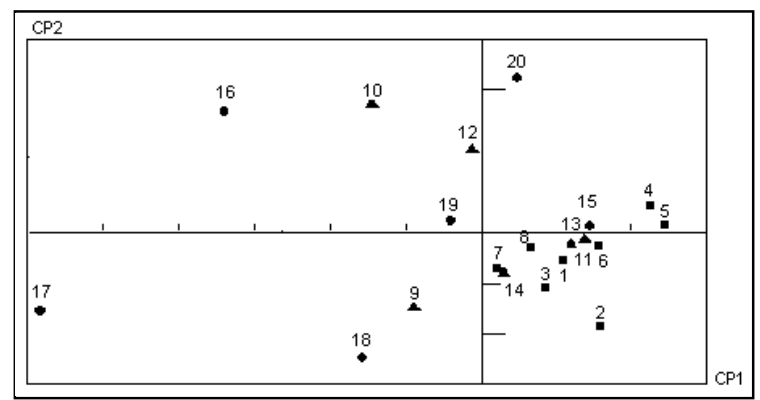

Figura 2 - Distribuição dos pontos correspondentes aos genótipos no plano definido pelos dois primeiros componentes principais para os descritores agronômicos.

Legenda: Virgínia $\bigcirc$ Spanish $\triangle$ Valência 


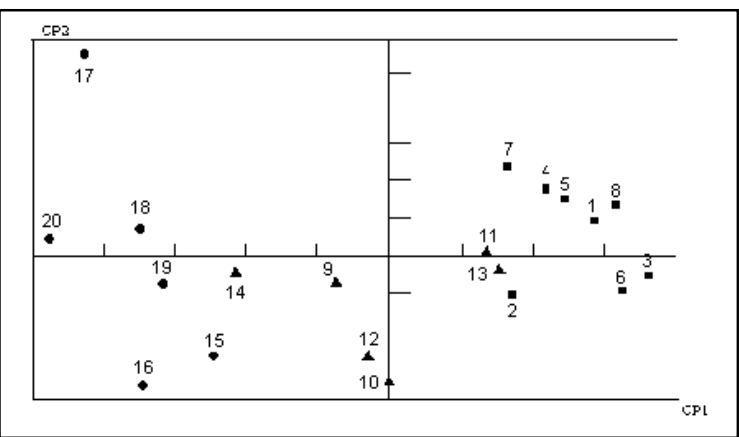

Figura 3 - Distribuição dos pontos correspondentes aos genótipos no plano definido pelos dois primeiros componentes principais para os descritores morfológicos e agronômicos.

Legenda: Virgínia

Spanish

Valência:

nos tipos agromorfológicos deva corresponder com a encontrada na base dos sistemas protéicoenzimáticos. Esse fato reforça a assertiva da maior estabilidade desses tipos e como tal explica a melhor descriminação dos mesmos com todos os descritores utilizados. A explicação para o comportamento exibido pelos tipos Valência pode residir no fato de que a maioria dos genótipos utilizados nesse estudo é constituída de tipos locais em poder dos agricultores. Dessa forma, podem ter sofrido menor pressão de seleção por parte dos melhoristas e com isso, ter preservado sua identidade genética em frente aos caracteres desse estudo. HAMOM \& TOURE (1990) trabalharam com cultivares de inhame pertencentes ao complexo cayanensis-rotundata e encontraram estreita correspondência entre os padrões do polimorfismo morfológico e enzimático. Verificaram, contudo, que em poucos casos, o primeiro não esteve associado à variação isoenzimática, o que, a propósito, foi também constatado no presente trabalho.

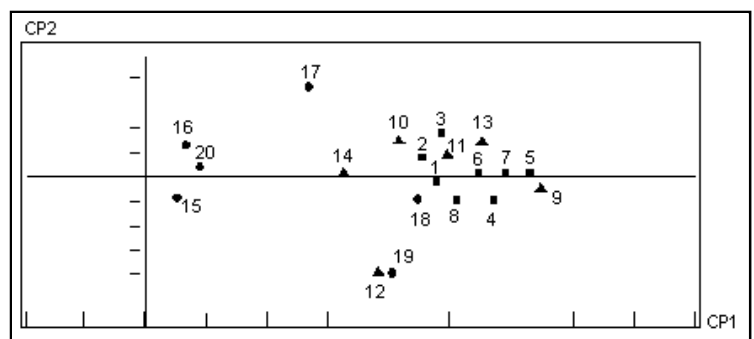

Figura 4 - Distribuição dos pontos correspondentes aos genótipos no plano definido pelos dois primeiros componentes principais para os descritores isoenzimáticos.

Legenda: Virgínia Spanish

\section{CONCLUSÃO}

Os descritores morfológicos: tipo botânico, pigmentação da haste principal, padrão de inflorescência, ponto de maturação das vagens, cor dos folíolos, hábito de crescimento, cor da semente e pilosidade da planta são de grande peso na classificação intra-específica no amendoim; os agronômicos de maior contribuição seletiva são: início da floração, peso de 100 sementes, número de vagens/planta, porcentagem de vagens chochas e rendimento em sementes; na combinação dos descritores agronômicos e morfológicos, os caracteres de maior carga são porcentagem de vagens chochas, número de semente/vagem, número de vagens/planta, rendimento em sementes, tamanho da vagem e o tipo botânico; no aspecto enzimático, nenhuma relação é observada entre os padrões de banda e os tipos botânicos; a maior dispersão entre os acessos ocorre entre os tipos Virgínia e Spanish, para os quais não se evidencia qualquer tendência de agrupamento na base dos descritores agronômicos adotados.

\section{REFERÊNCIAS BIBLIOGRÁFICAS}

ESQUIVEL, M., BARRIOS, M., WALÓN, L., et al. Peanut (Arachis hypogaea L.) genetic resources in Cuba. 1. Collecting and characterization. FAO/IBPGR Plant Genetic Resources Newsletters, Roma, v. 91/92, p. 9-15, 1993 a.

ESQUIVEL, M., FUNDORA, Z., HAMMER, K. Peanut (Arachis hypogaea L.) genetic resources in Cuba. 11 . Preliminary germoplasm evaluation. FAO/IBPGR Plant Genetic Resources Newsletters, Roma, v. 91/92, p. 17-20, 1993 b.

HAMOM, P., TOURE, B. Characterization of traditional yam varieties belonging to the Dioscorea cayanensis-rotundata complex by their isozymic patterns. Euphytica, Netherland, v. 48, p. 101-107, 1990.

KRAPOVICKAS, A. The origin, variability and spread of the groundnut (Arachis hypogaea L.). In: Ucko, P. J.; Folks, I. S. (Eds.). The domestication and exploration of plants and animals. London: Gerald Duckwortib, 1968. P. 427-442.

MEHNDIRATTA, P.D., PHUL, P.S., N.D. Genetic diversity in relation to fodder field and its components in Shorgum. Indian Journal of Genetic \& Plant Breeding, India, v. 31, n. 1, p. 300-306, 1970.

MOREIRA, J. de A,N., SANTOS, J.W. dos, OLIVEIRA, S.R. de $M$. Abordagens e metodologias para avaliação de germoplasma. EMBRAPA/CNPA; Brasilia: EMBRAPA-SPI, 1994, $115 \mathrm{p}$.

SANTOS, R.C., MOREIRA, J.A.N., CABRAL, E.L. Estudo da peroxidase na fenologia do amendoim submetido a estresse hídrico. Oleaginosas e Fibrosas, Campina Grande, v. 1, n. 1, p. 117-124, 1997a.

SANTOS, R.C. dos, MOREIRA, J. de A.N., ALMEIDA, R.P. de, et al. Caracterização e avaliação de germoplasma exótico e cultivares de Arachis hypogaea L. Campina Grande: Embrapa Algodão, 1997b, 20 p. (Documentos, n.43). 